\title{
Topological and Historical Considerations for Infectious Disease Transmission among Injecting Drug Users in Bushwick, Brooklyn (USA)
}

\author{
Kirk Dombrowski ${ }^{1 *}$, Richard Curtis ${ }^{1}$, Samuel Friedman ${ }^{2}$, Bilal Khan ${ }^{1}$ \\ ${ }^{1}$ Social Networks Research Group, John Jay College CUNY, New York, USA; ${ }^{2}$ National Development Research Institute, New York, \\ USA. \\ Email: *kdombrowski@jjay.cuny.edu
}

Received December $13^{\text {th }}, 2012$; revised January $22^{\text {nd }}, 2013$; accepted January $31^{\text {st }}, 2013$

\begin{abstract}
Recent interest by physicists in social networks and disease transmission factors has prompted debate over the topology of degree distributions in sexual networks. Social network researchers have been critical of "scale-free" Barabasi-Albert approaches, and largely rejected the preferential attachment, "rich-get-richer" assumptions that underlie that model. Instead, research on sexual networks has pointed to the importance of homophily and local sexual norms in dictating degree distributions, and thus disease transmission thresholds. Injecting Drug User (IDU) network topologies may differ from the emerging models of sexual networks, however. Degree distribution analysis of a Brooklyn, NY, IDU network indicates a different topology than the spanning tree configurations discussed for sexual networks, instead featuring comparatively short cycles and high concurrency. Our findings suggest that IDU networks do in some ways conform to a "scale-free" topology, and thus may represent "reservoirs" of potential infection despite seemingly low transmission thresholds.
\end{abstract}

Keywords: Social Network Analysis; Injecting Drug Users; Scale-Free Networks

\section{Introduction}

Recent interest by physicists in social networks and their relationship with disease transmission factors has prompted debate among social network theorists over the extent to which social networks conform more closely to general structural principles or to local social norms. This paper examines a smaller subset of this debate, and asks whether Injecting Drug User (IDU) networks responsible for the transmission of sexually transmitted and bloodborne diseases look more like topologies recently labeled "scale-free" by network theorists [1], or whether they are more like sexual networks, which have been shown to conform mainly to social principles like homophily and other specifically local norms [2]. This is important because the answer to this question bears strongly on intervention, prevention, and treatment strategies as they apply to IDU networks, and, to the extent that IDU networks continue to overlap with larger society in critical health related ways, bears on larger questions of public health and disease transmission more generally [3].

What follows is a reanalysis of data collected between 1991 and 1993 in the Bushwick neighborhood of Brook-

*Corresponding author. lyn, New York, on the social networks of injecting drug users (IDUs) [4]. Our analysis employs analytical strategies developed in the last few years by physicists working on what have come to be called "scale-free" networks, as developed by Barabasi and Albert [5,6]. Partly because our data were not collected with such an analysis in mind, throughout the paper we pose the findings and conclusions as "suggested," noting that several problems preclude more firm conclusions, and likewise note that this comparison leaves many aspects of IDU networks unexplained. Yet the apparent similarities between the network structure of the Bushwick IDU network and structures examined by Barabasi, Albert and others, prompt us to conclude that scale-free modeling can help elicit critical differences between IDU network topologies and those associated with sexual networks. Our results indicate that IDU networks may in fact represent the sorts of structural reservoirs of HIV and other bloodborne and sexually transmitted diseases at issue in the debates between physicists and sociologists, and thus require special consideration for prevention/transmission/ intervention planners. What's more, if IDU networks do in fact conform more closely to general structural principles than to local norms, police intervention strategies of 
the early 1990s employed in New York to suppress IDU networks ironically may have contributed to stronger network integration and thus potentially greater risk of disease transmission and network robustness. These considerations are discussed in more detail below.

\section{Sexual, IDU, and "Scale-Free" Networks}

Scale-Free modeling has, in last decade, been developed for a number of existing, real world networks, from the World-Wide-Web [7], to academic citation patterns, to the research partnerships of large pharmaceutical companies [8,9]. It has also been proposed for disease networks $[10,11]$, but here it has been critically received by many social network theorists [12]. Analytical work on sexual networks by social network researchers has more recently tended to reject purely structural models in favor of models that feature homophily and local norms as the main organizing principles [2]; though see $[13,14]$. Statistical tests on existing sexual network data sets by Jones, Handcock, and Holland have found that physicists' models do not meet "best-fit maximum likelihood estimates" for many short term sexual networks [12], which tend toward other sorts of distributions. In multi-model tests, scale free models seem to fit best those portions of sexual network topology with high levels of connectivity $[12,13,15]$, and less well for low degree nodes (i.e. those with few sexual partners). Further, in cases where scale free models do fit well, the degree distributions of those networks are quite different from most naturally occurring scale free networks, such as those discussed by Barabasi and others. Likewise, Bearman, Moody and Stovel [2] have shown that sexual networks lack the high levels of concurrence characteristic of, say, the Internet or the World-Wide-Web, showing instead that sexual networks look more like "spanning tree" graphs, with few (usually quite long) cycles.

IDU networks, however, differ from sexual networks in important ways, as they usually involve high concurrency, and short cycles. This is mainly due to the presence of a demonstrable "core" of highly connected users [16-18], producing short cycles and marked differences in edge degree value between core and periphery. This suggests that IDU networks, even where they contain sexual behavior among users, may operate according to different organizing principles than ordinary sexual networks, and thus contain different epidemiological dynamics. The specific features of those epidemiological dynamics go beyond the purpose of the current paper, though the influence of network cores on such issues as HIV prevalence and transmission rates has been explored in previous published work based on the Bushwick IDU network $[4,16,17]$. Here we propose only an examination of the degree distribution of the Bushwick IDU network, with the intention of showing that IDU networks differ from sexual networks in regard to the types of topological features often associated with disease transmission.

\subsection{The SFHR Data}

The collection and analysis of the data in the Social Factors for HIV Risk (SFHR) study is presented in much more detail in Friedman et al. Social Networks, Drug Injectors' Lives, and HIV/AIDS [4]. The data resulted from sociological, ethnographic, and epidemiological research into the drug and sexually related behaviors among drug injectors, as well as HIV and Hepatitis B exposure patterns for 767 injecting drug users in the Bushwick area of Brooklyn NY. Data were gathered over the course of 2 plus years, and analyzed in the book mentioned above and a number of related articles. Several social network papers were published as a result of this research, which firmly demonstrate the importance of network analysis among injecting drug users [16,17]. A social network diagram of the dyadic (person-to-person) links elicited in the Bushwick network is available in Figure 1. These links indicate contact via one or more "risk" behaviors: specifically, those respondents who injected drugs together or who engaged in sex with each other in the context of drug use in the thirty days prior to the interview. In prior analyses of this data, degree distribution was not systematically examined.

For purposes of the current paper, several areas of the SFHR study data collection are of principal concern. During the survey portion of the study, respondents were asked to estimate the number of people they had injected drugs with over the last 30 days. This was coded as INJP (for "injection partners") in the study. The responses here varied considerably, with many respondents giving round number figures and very rough estimates, and known hubs sometimes giving very low estimates. This is not surprising. Limitations of data gathered by "global item" questions and ego-centered networks are well known [19], and here are likely magnified by the illegal and socially stigmatized nature of some of the behaviors involved. Thus to more accurately gage the injecting behaviors beyond those simply reported by respondents' initial estimates, an additional network measure of respondent out-degree links was analyzed. Respondents were asked for a list of twenty close associates and whether the respondent had injected drugs or engaged in sex with that particular person in the last 30 days [20,21]. From these data, in-degree figures could also be calculated for each respondent, based on the number of persons in the study who named the subject as a recent partner. These network data created a dyad-based network picture which served as a partial check on the INJP estimates. Arc directions in Figure 1 indicate the direction 


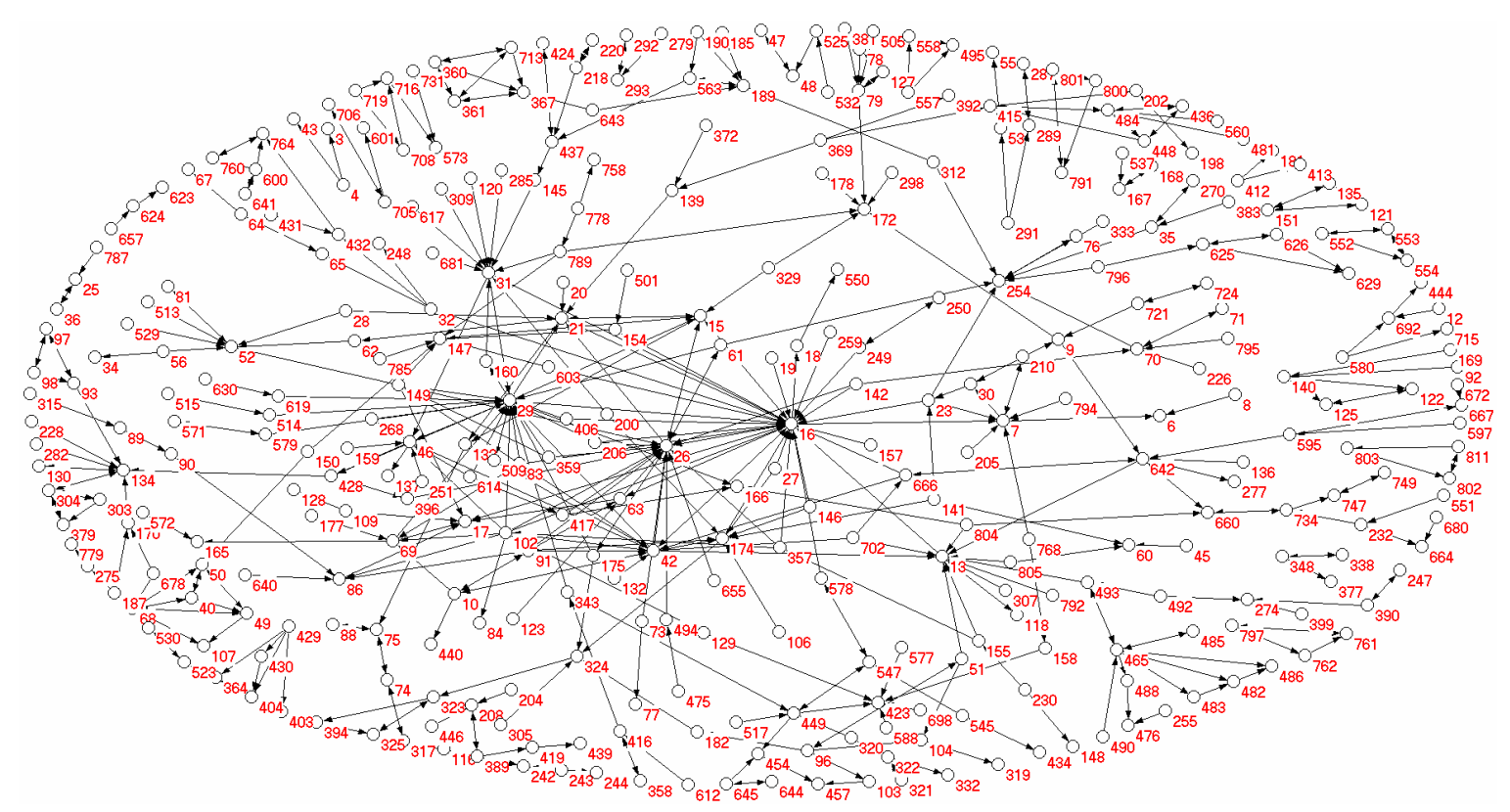

Figure 1. The risk network of injecting drug users discovered by the SFHR project in Bushwick, Brooklyn (USA) 1991-1993.

of the reports able to be verified by research staff from the list of study respondents [20]. Names of those outside of the study group or whose identities could not be verified are not included in this diagram or the dyad data discussed below.

Where partners could be identified, the network interview process thus produced data on two kinds of social links or "dyads." The first are "out-directed" arcs (coded "DyadAC"), where the interviewee, person A, identifies person $\mathrm{C}$ as a recent injecting/sex partner. One can thus visualize the report creating a directed arc from $\mathrm{A}$ to $\mathrm{C}$. Yet this same information also creates an in-directed arc or dyad connection (coded "DyadCA") for person C (the "receiver", if you will, of the injection/sex partnering reported by person A). One would expect that, when interviewed, the indicated partner, "C" would then name the original source, "A" as an injection partner as well. In that case, out-directed arcs revealed in the network interview by one respondent would be matched by in-directed arcs from others. Examples of these sorts of linkages are apparent in Figure 1. In a situation of full disclosure, simultaneous interviews, and $100 \%$ research effectiveness, in-degree and out-degree values ( $\Sigma$ DyadAC and $\Sigma$ DyadCA) would be identical in number for every respondent (and, ideally, would match the INJP number given by the respondent). This was, however, not always the case, particularly for network "hubs".

As mentioned above, some of the most widely cited injecting partners themselves gave low estimates of their number of partners (i.e. low INJP number), and identified few-sometimes zero-out-directed connections (i.e. a low $\Sigma$ DyadAC). Where these respondents tended to ap- pear in the network data was in other people's reports of their injecting partners (i.e. high numbers of in-di- rected connections, i.e. $\Sigma$ DyadCA). For this reason, it seemed critical to create a weighted average for each respondent of what would, under ideal conditions, consist of three identical figures (INJP, $\Sigma$ DyadAC, $\Sigma$ DyadCA; where INJP is, again, the number of self-reported injection partners, $\Sigma$ DyadAC is the number of people identified by respondent " $A$ " within the last 30 days as injection related partners - as far as could be identified as part of the study group by the research team - and $\Sigma$ DyadCA being the number of partnerings with " $A$ " reported by other respondents in the study group).

To compensate for the various problems described above, the three injector partner factors were combined to create a composite Mean Injection Total (MInjTot) for each of the 767 research respondents. That is, MInjTot figures were calculated for each individual (j) according to the following formula:

$$
\operatorname{MInjTot}_{j}=\frac{\mathrm{INJP}_{\mathrm{j}}+\overline{\mathrm{AC}_{\mathrm{j}}}+\overline{\mathrm{CA}_{\mathrm{j}}}}{3}
$$

where $\overline{\mathrm{AC}_{\mathrm{j}}}=\left(\left(\sum\right.\right.$ DyadAC $\left.\left._{\mathrm{j}}\right) \cdot \frac{\text { meanINJP }}{\text { meanDyadAC }}\right)$

and $\overline{\mathrm{CA}_{\mathrm{j}}}=\left(\left(\sum\right.\right.$ DyadCA $\left.\left._{\mathrm{j}}\right) \cdot \frac{\text { meanINJP }}{\text { meanDyadCA }}\right)$.

MInjTot $\mathrm{j}_{\mathrm{j}}$ is then rounded to the nearest integer value. This formula constitutes a weighted average of the three numbers $\left(\right.$ INJP $_{j}, \Sigma$ DyadAC $_{j}$, and $\Sigma$ DyadCA $_{j}$ ) for respondent " $\mathrm{j}$ ", using the ratio of the average of all initial 
total estimates (mean INJP) to the average of each dyad type (mean $\Sigma$ DyadAC and mean $\Sigma$ DyadCA) as the respective weighting factors. It should be noted, however, that this likely reflects an under-reporting of injection related partnering behaviors. The dyad research used only the names of 20 associates, resulting in an artificial ceiling of 20 for out-dyads (DyadAC) and, as such, a hidden but systemic constraint on DyadCA, especially for larger and medium sized hubs. As a result, for network hubs, both DyadCA and DyadAC (each of which would ideally equal the INJP estimate) will likely be lower than the INJP. A second factor is that, for some respondents, their involvement in other networks outside of the study area meant that those links would necessarily go unreported in the in-degree Dyad data, and thus their DyadCA estimate would be an undercount. The composite number, by including the dyad data, thus likely represents an under-reporting of network connectedness when those connections were to networks outside of Bushwick, as in-directed links include only those to others in the Bushwick network that could be identified as such by the research team. These factors necessarily minimize the firmness of the conclusions discussed below. Yet, as above, the justification for the creation of a composite MInjTot number was that, as suspected, the in-directed dyad data often produced levels of individual interconnectedness that went unreported or underreported in both the out-directed dyad data and INJP respondent estimates.

For purposes of the following model, injectors were treated as individual nodes, $j$, while an event of injection/sex partnering was considered a link, and the total number of links for each respondent, $k_{j}$, calculated according the MInjTot formula given above. As will be seen below, $k_{j}$ numbers ranged from 0 to 35 , with highest numbers of respondents having low numbers of links (a mode of 2). Respondents were then sorted by their respective number of links $k_{j}$ and the total number of respondents, $n_{i}$, for each respective $k$ value was determined. The number of respondents $n_{i}$ for a given number of links $k_{i}$ are given in Figure 2.

\subsection{Scale-Free Networks}

The first widely recognized paper on scale-free networks by Barabasi and Albert appeared in 1999 [6], with a subsequent full mathematical treatment and review of subsequent findings in 2002 [1]. In their original research on the World-Wide-Web (WWW), they found that the distribution of links ("arcs") between webpages ("vertices") seems to follow a "power law distribution." Such a distribution, they noted, is very different from those conforming to Poisson curves or random graphs, upon which much prior network modeling depended, and this obser-

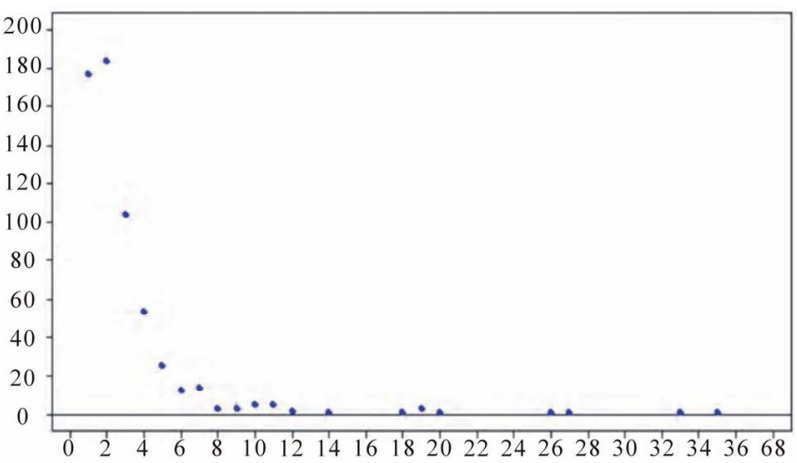

Figure 2. Number of users $\boldsymbol{n}$ (y-axis) with composite MInjTot, number of partners $k$ ( $x$-axis).

vation encouraged them to consider whether something other than a largely random, statistically normal distribution of connections was at work in this network. Subsequent research revealed many naturally occurring networks with apparent power law degree distributions, similar to the WWW, further prompting Barabasi and Albert to wonder whether identical, unusual network dynamics were responsible for a range of naturally occurring networks.

In graphs conforming to power law distributions, it is possible to calculate a reasonably accurate measure of the distribution of the connectedness of the various nodes/vertices in the network. As above, this is referred to as the "degree distribution," and it amounts to a graph of the number of nodes/vertices with the specified number of connections. On such a graph it is possible to calculate the "decay coefficient" of the graph, in effect a measure of the differential interconnectedness of the various nodes in the network. In the group of naturally occurring networks identified by Barabasi and Albert, networks exhibited recurring decay coefficients at or around $2[1]$.

To account for the regularity of this finding, Barabasi and Albert proposed a model of self-organizing networks where new nodes are added through time according to a linear preferential attachment or "rich-get-richer" strategy $[1,5,6,9]$, meaning that new nodes entering the network establish links to existing nodes in linear proportion to the number of links the latter already possess. In computer simulations of growing networks, Barabasi and Albert found that after a short time, linear preferential attachment strategies achieved steady decay coefficients between 2 and 3, and that the addition of new nodes after that point did not alter the shape (or "topology") of the graph, at least as far as the value of the decay coefficient $(\lambda)$ was concerned. This ability, to grow steadily without changing their topology, prompted Barabasi and Albert to label these sorts of networks "scale-free," to highlight the fact that they achieved topological stability regardless 
of their network size or the addition of new nodes and links, provided the rules of linear preferential attachment held steady.

Such theoretical networks demonstrate a number of characteristics in common with naturally occurring networks beyond sharing decay coefficient values around 2 . The first is that they are relatively impervious to error or failure - meaning that the removal of a high number of randomly selected nodes does not affect the topology of the network (or the value of the decay coefficient). This feature is referred to as network "robustness." Yet they also demonstrate "vulnerability to attack", such that network connectivity will fail (and the decay coefficient cross over a threshold into a dispersed and poorly integrated network) when a relatively low proportion of nodes are removed from the network, provided those taken out were "hubs" (i.e. nodes with high numbers of links). And finally, scale free networks exhibit "small world" characteristics [22], meaning that the shortest path between any two nodes turns out to be quite small, even in very large networks.

Based on these characteristics, a number of researchers $[7,10,11,13,14,23]$ argued that if sexual networks and other infection networks in general conformed to a scale-free distribution, then sexually transmitted diseases could persist in sexual network populations despite vanishingly small transmission thresholds due to the presence of a small but significant population with high numbers of links (i.e. high numbers of partners at risk for infection). This would be an effect of network robustness inherent in the structure of the network. Likewise, May and Lloyd [24] point out that the risk associated with membership in the network could be lowered dramatically not by the removal of large numbers of participants, but rather by focusing on altering the behavior of "hubs," i.e. those with the greatest number of partners (i.e. taking advantage of the network's "vulnerability to attack"). And finally, scale free theorists in general have pointed out that the small world character of these networks would mean that stemming infection in one segment of the population would meet with little lasting success, as re-infection remained only a few short network steps away, due to the nearness of many members of the network to many others [11]. As above, there is considerable debate about these findings as they apply to sexual networks, and our purpose is not to address this issue directly, but rather to ask whether the Bushwick IDU network conforms to a scale free topology, where such issues may apply more directly. If so, then many of the conclusions applied by scale free theorists to sexual networks as a whole might better apply to IDU networks instead.

The process of establishing the decay coefficient for a naturally occurring network (like the WWW) involves sorting nodes by the number of links they possess, then counting the number of nodes/vertices with a given number of links/edges. If we call $n_{i}$ the number of nodes in the network with $k_{i}$ links, the decay coefficient $\lambda$ of the particular network can then be ascertained by plotting the Log of $n$ versus the $\log$ of $k$ for all values of $i$ above 0 , and taking the slope of the resulting line...or in other words:

If $P(k) \sim k^{-\lambda}$ in a network where the highest number of links for any node is $\mathrm{t}$, then

$$
\lambda=-\left(\Delta \log n_{i} / \Delta \log k_{i}\right) \text { for } k_{1}, k_{2}, k_{3} \cdots k_{t}
$$

where $n_{i}=$ number of nodes with edges $k_{i}$. The slope of the line in a plot of $\log \mathrm{n}$ versus $\log \mathrm{k}$ is thus the decay coefficient $\lambda$, which represents, again, a formal measure of the degree distribution of the network.

\section{Results}

Looking back at Figure 2, we note the apparent power law distribution of the data. A $\log / \log$ (base 10) graph of the data from Figure 2 is available in Figure 3, and the regression line of this data is indicated on the graph. As discussed above, its slope is the decay coefficient $\lambda$ for the degree distribution in Figure 2. In this graph, $\lambda=1.8$ (with a Pearson's $r$ value of 0.947 , and an adjusted $r$ square value of 0.891).

From this graph we note that MInjTot figures conform well to a log linear representation: the Pearson's $r$ value indicating a close association of the points. A second immediate observation is that the value for $\lambda=1.8$ is close to that discovered for a host of real-world networks by Barabasi, Albert and others: comparable decay coefficients were found for networks such as Hollywood actor collaborations $\lambda_{\text {actor }}=2.3$, the World-Wide-Web $\lambda_{\text {www-in }}=$ $2.1, \lambda_{\text {www-out }}=2.4$, the metabolic network of E.coli bacteria $\lambda_{\text {ecoli }}=2.2$, Internet Domains $\lambda_{\text {domain }}=2.1$, phone calls $\lambda_{\text {phone }}=2.1$, Co-authorship among neurologists $\lambda_{\text {neuro }}=$ 2.1 , or mathematicians $\lambda_{\text {math }}=2.5$, or other academics

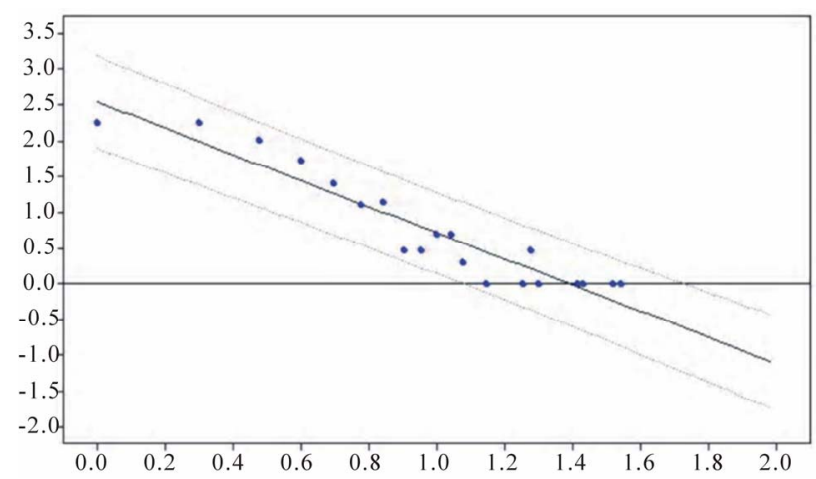

Figure 3. Log/Log (base 10) graph of Figure 2. 
$\lambda_{\text {spires }}=1.2[1$, p. 51$]$. It is also worth noting that the value is well below that normally discussed for sexual networks, i.e. where $\lambda$ usually equals somewhere between 3 and $4[10,14,15]$. This will be discussed in greater detail below. Equally clear is the "hubbed" nature of the network, with wide differences in the distribution of links among nodes, and a significant population having many times the average $\left(k_{\text {avg }}=3.0\right)$ and modal $\left(k_{\text {mode }}=2\right)$ number of links. Indeed, in Figure 2, 42 of the 767 respondents (5\% of the total network) accounted for $508 /$ 1789 (about 28\%) of the total links in the network, with a range of $k$-values from 7 to 35 .

A comparison with previously published ethnographic and social network analyses of this same population helps establish the representativeness of MInjTot formula. Ethnographic observation indicates that the three most "linked" individuals according to the MInjTot formula are identifiable respondents who show up as central hubs in a social network of "core" participants compiled from observation data (described fully in [18]) and from social network "Seidman k-core" analyses of this same population $[4,16]$. In Figure 3, the most linked respondents, respectively from right to left (high to low) on the x-axis, correspond to respondents with identification numbers 16 , 29, and 91 in Figure 1, with respondents 7, 10, 13, 21, $23,26,31,42,46,83,102,210,259$ appearing in the top 25 most linked hubs according to the MInjTot formula as well. Many of these same individuals also appear in the ethnographic/observational core of this same network [18, p. 236] and are clear hubs in the network diagram of Figure 1 (again, drawn entirely from the dyad data). Indeed, the ethnographic research component of the original SFHR study identified respondents 16, 29 and 42 from Figure 1 as locally well-known shooting gallery operators and central players in the Bushwick drug scene [18, p. 235], a point we will return to below.

Network data on these and other individuals also shows the ability of the composite MInjTot formula to respond to a variety of reporting scenarios: respondent 16 estimated a total of 35 partners in the last 30 days, identifying 6 other members of the study (DyadAC) and being identified as such by 28 other study participants (DyadCA), and respondent 29 estimated 30 partners, identified 8 (DyadAC) and was identified by 19 (DyadCA). Yet respondents 26 and 42, each gave self-reports of low numbers of partners (8 and 1 respectively), which masked high in-degree values (17 and 26 in-degree values, respectively). They also appear in the model as highly connected hubs in Figures $\mathbf{2}$ and 3. And finally, in a somewhat opposite case, respondent 91's estimate of 84 injection/sex partners was not matched by high DyadAC (6) or CA (1), which may indicate a preponderance of partners outside of the Bushwick network, or removal from the network during the study period or a desire to impress the interviewer with his status as an important person in the network. As a result, this individual appears with higher overall degree in Figures $\mathbf{2}$ and $\mathbf{3}$ than indicated in Figure 1, but not as high as she would be in a graph based solely on self-reported INJP figures. Altogether, the composite formula was able to identify key players in the network despite high variability in reporting profiles, and produce network structures borne out by prior network analysis and ethnographic observation.

\section{Discussion}

With the representativeness of the graph established and its similarities to other naturally occurring scale free networks apparent, part of what must be addressed is what is achieved by doing a "scale-free" analysis, as this is often misperceived (and misadvertised). The model above might be termed a "scale-free analysis" in the following sense; it is an analysis of the degree distribution of a network that shows it to conform to the type of degree distribution that Barabasi and Albert found could be reproduced by means of simple, linear preferential attachment. For the Bushwick IDU network, this is not surprising at a very general level, given that IDU network members ordinarily become members of a particular injector network via someone who was already part of it. Indeed, ethnographic observation points out that some of the more central figures in the Bushwick network, apart from shooting gallery operators, were individuals who functioned as connectors between core members and new or more marginal network members [18].

While the purpose of such a comparison with Barabasi-Albert degree distributions is primarily suggestive, and while degree distribution graphs are only one measure of network topology (and many other network parameter measures are possible: see [16] for Seidman kcore distribution analysis of this same population), nevertheless, the suggestion that IDU networks contain preferential attachment dynamics is important, as it differentiates them from findings about sexual networks [2], and implicates them in important disease transmission dynamics $[10,13,14]$. Past research demonstrates the existence of a core-periphery structure, short cycles, and high concurrency in the Bushwick IDU network $[4,16]$, all of which contrast strongly with the spanning tree models of sexual networks described by Bearman, Moody and Stoval. Such characteristics indicate that the "small world" characteristics typical of scale free networks could very well be present in the Bushwick IDU network. This is critical as injection sharing and sexual contact associated with injecting drug networks are already known as important vectors for disease transmission, further indicating that unique transmission dynamics may 
take place among them that deserve serious attention [3]. As above, in small world networks, the network topology itself can be responsible for preserving a high level of system-wide risk even when disease prevalence is reduced to near zero levels.

The decay coefficient for the Bushwick IDU network is interesting in this way as well. This is, as far as we know, one of few cases in the disease transmission literature where the power-law decay coefficient of a disease transmission network is at or below 2. In contrast, sexual networks usually demonstrate decay coefficients of three or more [10,13-15]. May and Lloyd [24] have shown mathematically that in disease transmission networks, topologies with decay co-efficient values below 3 indicate that there will always be someone in the population-with enough contacts to spread the disease, independent of population-wide disease transmission probabilities. The possibility that IDU networks represent such a group would then require special attention, confirming conclusions suggested by other analyses of this data as well [25] that unique dynamics related to network structure are at work. Indeed, from these graphs there is a reasonable suggestion that IDU networks represent the sorts of reservoirs of infection hypothesized by scale-free theorists.

That being said, it is important to note that, like sexual networks, IDU networks do nevertheless conform to local norms of risk behavior [4] and particular local events. Yet these too must be understood to take place within a specific structural framework. Evidence of this may be present in Figure 3 as well. Of particular interest is that, in Figure 3, the distribution of data points in relation to the best fit line shows a clear deviation pattern. In Figure 3 , those points to the left of the midpoint value of the $\mathrm{x}$-axis $(\log k=0.8)$ tend to be above the regression line, while those to the right of the midpoint value tend to be below it, with the exceptions of a few points at the highest $\mathrm{k}$ values. This would indicate that the decay coefficient of 1.8 is in fact lowered by the several points at the very far right (the highest values) on the $\mathrm{x}$-axis, which stretch the $\mathrm{x}$-intercept of the regression line to the right, and lessen its slope. At a superficial level, this point bears out the claim by scale-free and small world theorists (see [22]) that, in networks with highly differential levels of connectedness, a few individuals with high numbers of links (in Figure 3, this is four individuals) can significantly alter the topology of the network.

As importantly, however, this aspect of the topology of Figure 3 indicates that a few individuals at the far right side of the graph represent a level of individual interconnectedness $k_{j}$ above that predicted by a "scale-free" distribution. When graphs of the model were repeated without these 4 points, they return a decay coefficient of $\lambda=2.1$, a near perfect topological match for the scale free model. Given this, the source of the deviation of these four points (again, representing respondents 16, 29, 91, and 162 in Figure 1) from the ideal-typical scale free model is of particular interest. One central reason for this deviation is suggested by ethnographic and interview data, which points to changes in the Bushwick drug market at the time of the study. During the 1991-1993 time frame, New York City as a whole [26], and Bushwick in particular, were undergoing a change in drug interdiction regimes aimed at closing down "open air" drug markets and injection locations [27]. This strategy involved broad "sweeps" in which outdoor users were routinely arrested for small amounts of drug possession. As places serving as outdoor drug use locations were systematically pursued by law enforcement, outdoor drug use became increasingly precarious, and indoor, more discreet drug use locations (shooting galleries) grew in importance as a result $[28,29]$. Local estimates place them at 5 or 6 in Bushwick at any given time over the course of the two year study, and fluidity among locations was constant as interdiction turned toward these locations as well.

Ethnographic observations and interviews with respondents in the Bushwick study confirm that many of the major network nodes identified in Figures $\mathbf{2}$ and $\mathbf{3}$ by the composite MInjTot figure turned out to be either shooting gallery operators (again respondents 16, 26, 29, and 42), or close associates of these same individuals who served as injection specialists or good vein locators, for example, or runners for various kinds of equipment, or recruiters for gallery operators (which included respondents 7, 13, 31, 46, 91, 102), most of whom are identifiable in Figure 1, and figure significantly as "hubs" in Figures 2 and 3. The exaggerated centrality of high-end hubs suggests to us that police interdiction strategies may have strengthened the hub-like nature of gallery operators, as network hubs found their centrality enhanced by significant numbers of clients in search of safe injection locales. This was particularly true for Hispanic gallery operators, whose greater tie-in to the local community made the re-establishing of gallery locations and reconstruction of a network of users both more likely and quicker despite enforced mobility and occasional removal from the network [27]. Beyond this, those who remained within the Bushwick network across the entire study period by avoiding arrest and other complications were further able to accumulate high numbers of links.

As suggested by the data in Figure 3, then, interdiction/enforcement strategies may thus have boosted the level of interconnectedness for gallery operators and associated hubs beyond that predicted by self-organizing, scale-free models, increasing network interconnectedness 
and pushing the network as a whole toward a more "fat-tailed" distribution.

Without comparable topological data for the periods before or after 1991-1993 study period and a period of peak police activity, observations like these are mostly speculative. Never the less, by further heightening the "hubbed" nature of the network, enforcement strategies arguably increased the "small world" nature of the network (shortening network distances across which infections pass), and the robustness of the network itself - meaning that police "sweeps" of users were further rendered moot in terms of disrupting the network structure and interconnectedness.

\section{Remaining Questions}

As a final comment, it is also worth repeating that preferential attachment, Barabasi-Albert models do not account for new links created by already established network actors to others also already in the network. Nor do they account for the effects of particular nodes disappearing from the network. Do their former partners go on to form new links? If so, to whom? Does preferential attachment hold in aging networks (initial evidence is that it does not; see Albert and Barabasi [1]? These are critical features of IDU networks that likely also require different sorts of attachment models, and likely quite different sorts of network parameterization to detect.

Despite this, what remains critical here is the possibility that IDU networks conform to a topology quite different from that of sexual networks, despite the fact that they remain linked to sexual networks (and thus represent areas of significant risk to the non-IDU population $[3,25])$. The topological findings demonstrated here extend those already discussed for the SFHR data, and argue once again for special attention and special understanding of IDU networks and their structural (as well as behavioral) uniqueness, and question the viability and impact of interdiction strategies that do not address structural dynamics [30].

\section{Acknowledgements}

Support for the original data collection was provided by the National Institute on Drug Abuse grant DA06723 "Social Factors and HIV Risk" and grant P30DA11041 "Center for Drug Use and HIV Research." The New York State Department of Health AIDS Institute assisted in drawing serum samples, HIV testing, and referrals. The views expressed herein do not necessarily reflect the positions of these institutes. This project was also supported by NIH/NIDA Challenge Grant 1RC1DA02847601/02 awarded to the CUNY Research Foundation and John Jay College, CUNY. The opinions, findings, and conclusions or recommendations expressed in this publication are those of the authors and do not necessarily reflect those of the National Institute of Health/National Institute on Drug Abuse.

\section{REFERENCES}

[1] R. Albert and A.-L. Barabasi, "Statistical Mechanics of Complex Networks," Reviews of Modern Physics, Vol. 74, No. 1, 2002, pp. 47-97. doi:10.1103/RevModPhys.74.47

[2] P. S. Bearman, J. Moody and K. Stovel, "Chains of Affection: The Structure of Adolescent Romantic and Sexual Networks," American Journal of Sociology, Vol. 110, No. 1, 2004, pp. 44-91. doi:10.1086/386272

[3] S. R. Friedman, M. Bolyard, P. Mateu-Gelabert, P. Goltzman, M. P. Pawlowicz, D. Z. Singh, G. Touze, D. Rossi, C. Maslow, M. Sandoval and P. L. Flom, "Some Data-Driven Reflections on Priorities in AIDS Network Research," AIDS and Behavior, Vol. 11, No. 5, 2007, pp. 641-651.

[4] S. R. Friedman, R. Curtis, A. Neaigus, B. Jose and D. C. Des Jarlais, "Social Networks, Drug Injectors' Lives, and HIV/AIDS," Kluwer Academic/Plenum Publishers, New York, 1999.

[5] R. Albert, H. Jeong and A.-L. Barabasi, "Error and Attack Tolerance of Complex Networks," Nature, Vol. 406, No. 6794, 2000, pp. 378-382. doi:10.1038/35019019

[6] A.-L. Barabasi and R. Albert, "Emergence of Scaling in Random Networks," Science, Vol. 286, No. 5439, 1999, pp. 509-512. doi:10.1126/science.286.5439.509

[7] Z. Dezso and A.-L. Barabasi, "Halting Viruses in ScaleFree Networks," Physical Review E, Vol. 65, No. 5, 2002, Article ID: 055103(R). doi:10.1103/PhysRevE.65.055103

[8] S. N. Dorogovtsev and J. F. F. Mendes, "Evolution of Networks: From Biological Nets to the Internet and WWW," Oxford University Press, New York, 2003.

[9] A.-L. Barabasi, "Linked," Plume Books, New York, 2003.

[10] F. Liljeros, C. R. Edling, L. A. N. Amaral, H. E. Stanley and Y. Aberg, "The Web of Human Sexual Contacts," Nature, Vol. 411, 2001, pp. 907-908. doi: $10.1038 / 35082140$

[11] M. E. J. Newman, "Spread of Epidemic Diseases on Networks," Physical Review E, Vol. 66, No. 1, 2002, Article ID: 016128. doi:10.1103/PhysRevE.66.016128

[12] J. H. Jones and M. S. Handcock, "Social Networks (Communication Arising): Sexual Contacts and Epidemic Thresholds," Nature, Vol. 423, No. 6940, 2003, pp. 605 606. doi: $10.1038 / 423605 \mathrm{a}$

[13] A. Schneeberger, R. Nat, C. Mercer, S. Greggson, N. Ferguson, C. Nyamukapa, R. Anderson, A. Johnson and G. Garnett, "Scale-Free Networks and Sexually Transmitted Diseases," Sexually Transmitted Diseases, Vol. 31, No. 6, 2004, pp. 380-387. doi:10.1097/00007435-200406000-00012

[14] F. Liljeros, C. R. Edling, H. E. Stanley, Y. Aberg and L. 
A. N. Amaral, "Social Networks (Communication Arising): Sexual Contacts and Epidemic Thresholds," Nature, Vol. 423, No. 6940, 2003, p. 606. doi:10.1038/423606a

[15] D. Hamilton and M. S. Handcock, "Degree Distributions of Sexual Networks: Should We Buy Scale Free?" Paper Presented at the International Sun Belt Social Network Conference, Vancouver, May 2006.

[16] S. R. Friedman, A. Neigus, B. Jose, R. Curtis, M. Goldstein, G. Ildefonso, R. B. Rothenberg and D. C. Des Jarlais, "Sociometric Risk Networks and Risk for HIV Infection," American Journal of Public Health, Vol. 87, No. 8, 1997, pp. 1289-1296. doi:10.2105/AJPH.87.8.1289

[17] S. R. Friedman, B. J. Kottiri, A. Neaigus, R. Curtis, S. H. Vermund and D. C. Des Jarlais, "Network-Related Mechanisms May Help Explain Long-Term HIV-1 Seroprevalence Levels That Remain High but Do Not Approach Population-Group Saturation," American Journal of Epidemiology, Vol. 152, No. 10, 2000, pp. 913-922. doi:10.1093/aje/152.10.913

[18] R. Curtis, S. R. Friedman, A. Neaigus, B. Jose, M. Goldstein and G. Ildefonso, "Street-Level Drug Markets: Network Structure and HIV Risk," Social Networks, Vol. 17, No. 3-4, 1995, pp. 229-249. doi:10.1016/0378-8733(95)00264-O

[19] P. V. Marsden, "Recent Developments in Network Measurement," In: P. Carrington, J. Scott and S. Wasserman, Eds., Models and Methods in Social Network Analysis, Cambridge University Press, Cambridge, 2005, pp. 8-30. doi:10.1017/CBO9780511811395.002

[20] G. Ildefonso, S. Friedman, R. Curtis, A. Neaigus, B. Jose, and D. C. Des Jarlais, "Chapter 13. Appendix: Methods for Assigning Linkages in Studies of Drug Injector Networks," In: S. Friedman, Ed., Social Networks, Drug Injectors' Lives, and HIV/AIDS, Kluwer Academic/Plenum Publishers, Berlin, 1999, pp. 239-254.

[21] A. Neigus, S. R. Friedman and M. Goldstein, "Using Dyadic Data for a Network Analysis of HIV Infection and Risk Behaviors among Injecting Drug Users," In: R. Needle, Ed., Social Networks, Drug Abuse and HIV Transmission, National Institute on Drug Abuse, Rockville, 1995, pp. 20-37.
[22] D. J. Watts, "Small Worlds," Princeton University Press, Princeton, 1999.

[23] R. Pastor-Satorras and A. Vespignani, "Epidemic Spreading in Scale-Free Networks," Physical Review Letters, Vol. 86, No. 14, 2001, pp. 3200-3203. doi:10.1103/PhysRevLett.86.3200

[24] R. M. May and A. L. Lloyd, "Infection Dynamics on Scale-Free Networks," Physical Review E, Vol. 64, No. 6, 2001, Article ID: 066112. doi:10.1103/PhysRevE.64.066112

[25] S. R. Friedman, P. L. Flom, B. J. Kottiri, J. Zenilman, R. Curtis, A. Neaigus, M. Sandoval, T. Quinn and D. C. Des Jarlais, "Drug Use Patterns and Infection with Sexually Transmissible Agents among Young Adults in a HighRisk Neighborhood in New York City," Addiction, Vol. 98, No. 2, 2003, pp. 159-169. doi:10.1046/j.1360-0443.2003.00271.x

[26] R. Curtis, "The Improbable Transformation of Inner-City Neighborhoods: Crime, Violence, Drugs and Youth in the 1990s," The Journal of Criminal Law and Criminology, Vol. 88, No. 4, 1998, pp. 1233-1276. doi: $10.2307 / 1144256$

[27] R. Curtis, "The War on Drugs in Brooklyn, New York: Street-Level Drug Markets and the Tactical Narcotics Team," Doctoral Dissertation, Columbia University, New York, 1996.

[28] R. Curtins and M. Sviridoff, "The Social Organization of Street-Level Drug Markets and Its Impact on the Displacement Effect," In: R. McNamara, Ed., Crime Displacement: The Other Side of Prevention, Cummings and Hathaway Publishers, New York, 1994, pp. 155-171.

[29] R. Curtis, "The Changing Drug Scene in Brooklyn, NY Neighborhoods," In: A. Karmen, Ed., Crime and Justice in New York City, McGraw Hill, New York, 1998.

[30] K. Dombrowski, B. Khan, K. McLean, R. Curtis, T. Wendel, E. Misshula and S. Friedman, "A Re-Examination of Connectivity Trends via Exponential Random Graph Modeling in Two IDU Risk Networks," Substance Use and Misuse, Vol. 49, 2013. 